\title{
A COMPARATIVE STUDY ON THE PERFORMANCE OF THE INSAR PHASE FILTERING APPROCHES IN THE SPATIAL AND THE WAVELET DOMAINS
}

\author{
Wajih Ben Abdallah ${ }^{1}$ and Riadh Abdelfattah ${ }^{1,2}$ \\ ${ }^{1}$ Higher School Of Communications of Tunis \\ University of Carthage, COSIM Lab \\ Route de Raoued KM 3.5, Cite El Ghazala Ariana 2083 \\ benabdallah.wajih@supcom.rnu.tn \\ ${ }^{2}$ Telecom Bretagne, Institut de Télécom, Département ITI \\ Technopôle Brest-Iroise CS 83818, 29238 Brest Cedex 3 - France \\ riadh.abdelfattah@supcom.rnu.tn
}

Commission III/3

KEY WORDS: WInPF, Lee filter, FAMM, WMF, Wavelet domain, Interferogram SAR.

\begin{abstract}
:
In this paper, we studied and tested different filtering approaches of the SAR interferograms in the spatial and wavelet domains. In the spatial domain, we applied the classic Lee filter and the Weighted Median Filter WMF. In the wavelet domain, we tested a noise reduction algorithm WInP proposed by López and Fàbregàs and its enhanced version FAMM developed by Abdelfattah and Bouzid. Those filters are validated with different SAR interferograms provided by Radarsat-2, Envisat, ERS-2 and COSMO-SkyMed SLC data acquired over regions of Mahdia and Ben Guerden in Tunisia. The aim of this study is to select the optimal filtering approach with respect to the fringe pattern in the interferogram. This selection is based on the Digital Elevation Model error computed between the filtered unwrapping phase image and the Global ASTER DEM of the same regions and verified with simulated interferograms.
\end{abstract}

\section{INTRODUCTION}

Interferometric Synthetic Aperture Radar (InSAR) is a technique used to measure the relief and detect any changes accrued to the surface of the earth by calculating the phase difference information between two complex radar images (Abdelfattah and Nicolas, 2002),(Goldestein et al., 1988). Indeed, a phase difference image is generated using both complex images, which is named interferogram. The phase difference is proportional to the geomorphological parameters (height, displacement,etc...) of the imaged terrain (Abdelfattah and Nicolas, 2002).

In practice the real interferogram is affected by an additive noise and various decorrelation effects (thermal noise, geometrical conditions,etc...). Several filters have been proposed, such as the median filters (Bezerra Candeias et al., 1995), filters based on the minimisation of the mean squared error (Fornaro and Guarnieri, 2002) and the Gaussian filters (Geudtner D. and R., 1994). Xie et al. (Xie and Pi, 2011) uses the unscented Kalman filter that allow the noise filtering and the phase unwrapping simultaneously. Unfortunately, they are not adapted to noises with local variations. As an alternative to overcome this drawback, the filters that use an average process and which require a locally phase unwrapping and then rewrapping step, in order to preserve phase discontinuities, are more adapted to SAR interferogram noise problem. Thus, the noise reduction operation is commonly applied before phase unwrapping process (Huot et al., 1998).

In this paper, we consider four different methodologies for denoising the interferogram: an adaptive and a median filter based on the spatial domain and two other approaches in the wavelet domain. They are respectively the filtering approach proposed by Lee (Lee et al., 1998), the weighted median filter presented in (Arce, 1998), the denoising algorithm given by (Lopez-Martinez and Fabregas, 2002) and the filtering method given in (Abdelfattah and Bouzid, 2008). All of these approaches are tested on real interferograms from pairs of SLC data produced by Radarsat-2,
Envisat, ERS-2 and COSMO-SkyMed satellites respectively over the region of Mahdia and Ben Guerden in Tunisia. This paper is organized as follows. First, the four filtering approaches are presented in section 2. Section 3 describes the used data. Section 4 presents the experimental results with quantitative and qualitative comparisons between these four filtering methods. The conclusion derived from this work is given in last section.

\section{INTERFEROGRAM FILTERING}

The Synthetic Aperture Radar systems can produce for each acquisition a complex values image (Goldestein et al., 1988). Then for two acquisitions the correlation coefficient $\gamma$ between the two complex images is computed. The corresponding phase $\varphi=\arg [\gamma]$ is called the interferometric phase. As any measure process, the interferometric phase is affected by a phase noise $n$.

\subsection{Interferogram Filtering In The Spatial Domain}

2.1.1 The Lee filter This filter is proposed in 1998 by Lee $e t$ al. (Lee et al., 1998). It assumes that the noise $n$ can be modeled as an additive white noise in the real domain:

$$
\phi=\varphi+n
$$

where $\phi$ and $\varphi$ are the observed and original phase respectively and $n$ is zero mean noise which depends on the number of look $k$ and the coherence value $|\gamma|$. The original phase and the noise are supposed to be independent from each other (Hess-Nielsen and Wickerhauser, 1996). The Lee filter can be applied in the real or complex phase plane respectively. In the real plane, the basic principle of the filter is to use 16 different directional masks to filter the noise along the fringes (Lee et al., 1998). The filtering process in this case is composed of three steps: The first step consists to unwrap the phase pixels in the operating $9 \times 9$ window. Then, each phase at pixel $(x, y)$ of window is compared with the 
Lee filter
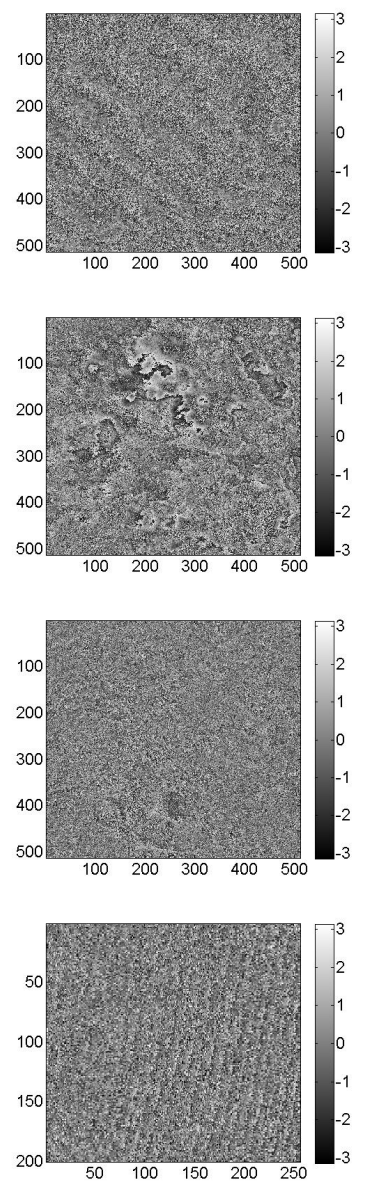

WM filter
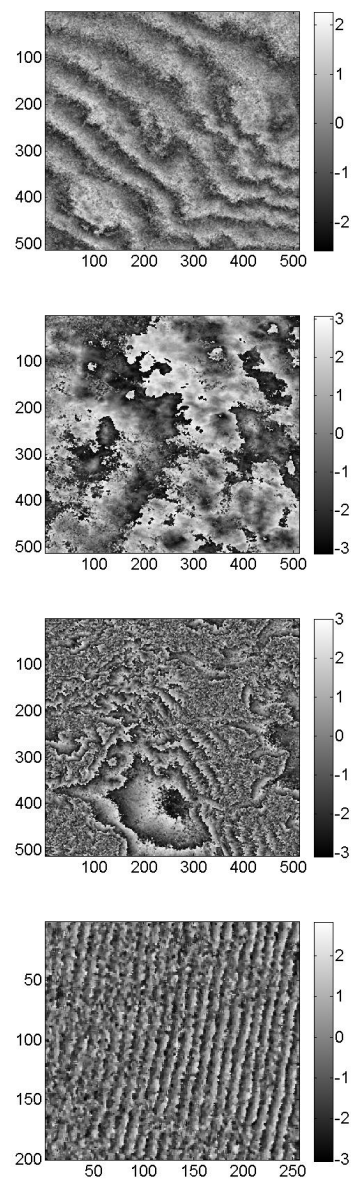

WInP filter
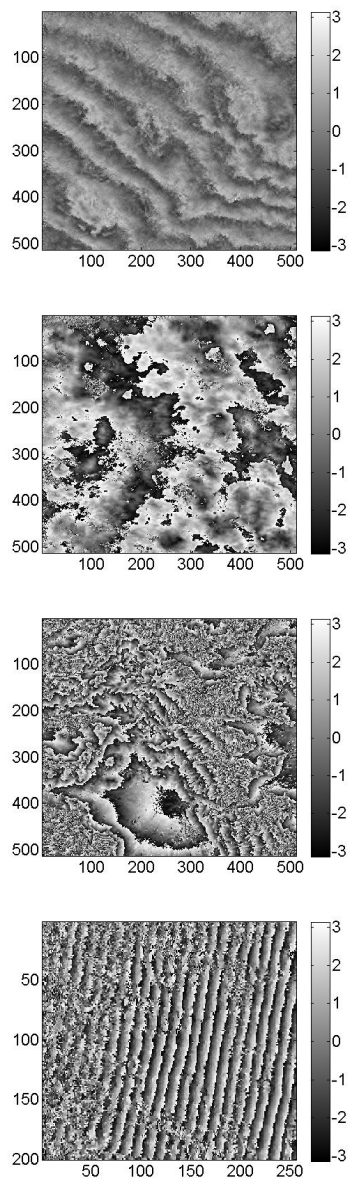

FAMM filter
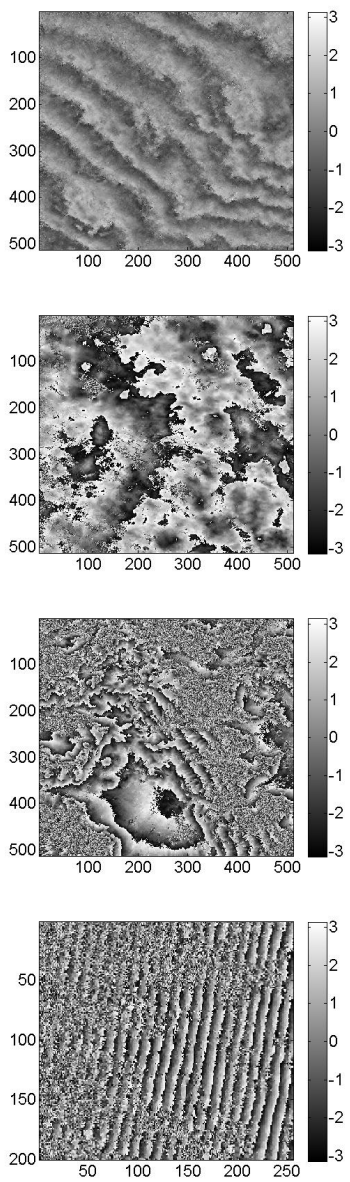

Figure 1: Filtering result of real interferograms generated from SLC data of the four satellites and acquired over the region of Mahdia : Radarsat-2 (first row) and Envisat (second row) and over the region of Ben Guerden : ERS-2 (third row) and COSMO-SkyMed (fourth row).

phase of the complex average $e^{j \phi}$ of the center window $3 \times 3$ and the unwrapped phase $\varphi_{u}$ is computed as follows:

$$
\varphi_{u}(x, y)=\left\{\begin{array}{l}
\varphi(x, y)-2 \pi \text { if } \varphi(x, y)>\arg \left[e^{j \phi}\right]+\pi \\
\varphi(x, y)+2 \pi \text { if } \varphi(x, y)<\arg \left[e^{j \phi}\right]-\pi
\end{array}\right.
$$

After filtering, the filtered phase has to be rewrapped. The next step is to select the directional window to be used to apply the filter, by computing the variances in the different 16 windows (Lee et al., 1998) and selecting the window with the minimum variance. The third step is to compute the phase noise standard deviation $\sigma_{n}$ using the lookup table created by a polynomial approximation for a specified number of looks (Lee et al., 1998).

2.1.2 The Weighted Median Filter WMF The Weighted Median Filter (Arce, 1998) is a variation of the median filter by weighting the values of the entry. If we consider an observation window $X=\left(X_{1}, \ldots, X_{N}\right)$ with size $N$ and if the weight of the particular filter are noted $\left(W_{1}, \ldots, W_{N}\right)$, then the output of the filter is calculated with the following algorithm :

Step 1: compute the threshold $T_{0}=1 / 2 \sum_{i=1}^{N}\left|W_{i}\right|$

Step 2: multiply the observation samples $X_{i}$ with their weights signs.

Step 3 : the observation samples are sorted from smaller to bigger.

Step 4 : calculate the corresponding absolute values of the sorted observation samples.

Step 5 : by summing the absolute weights, beginning with the maximum samples, the output is signed sample whose absolute weight causes the sum to become $\geq T_{0}$.

\subsection{Interferogram Filtering In The Wavelet Domain}

2.2.1 The WInP filter In (Lopez-Martinez and Fabregas, 2002), the noise model given at (1) is expressed in the complex domain to avoid the phase jumps in real domain which prevent the correct unwrapping of the interferometric phase (Abdelfattah and Bouzid, 2008). The observed phase is rewritten in the complex domain as follows:

$$
e^{j \phi}=\cos (\varphi+n)+j \sin (\varphi+n)
$$

To detect the noisy pixels in complex domain, López and Fàbregas (Lopez-Martinez and Fabregas, 2002) propose to use the wavelet decomposition which decomposes a signal into its low frequency components (approximations) and high frequency components (details) (Hess-Nielsen and Wickerhauser, 1996). Using the additive noise model of (3), (Lopez-Martinez and Fabregas, 2002) proposed to apply the three scales wavelet transform and the Discrete Packet Wavelet Transform (DPWT) at the third decomposition level to create an equivalent model. In this case, the wavelet transform not only applied on the low coefficients of the second decomposition but also on the other three high frequency bands. Then, 16 sub bands are obtained and containing mainly the signal coefficients. To each pixel in those 16 bands corresponds 48 pixels in the three high frequency bands of the first transform 
Lee filter
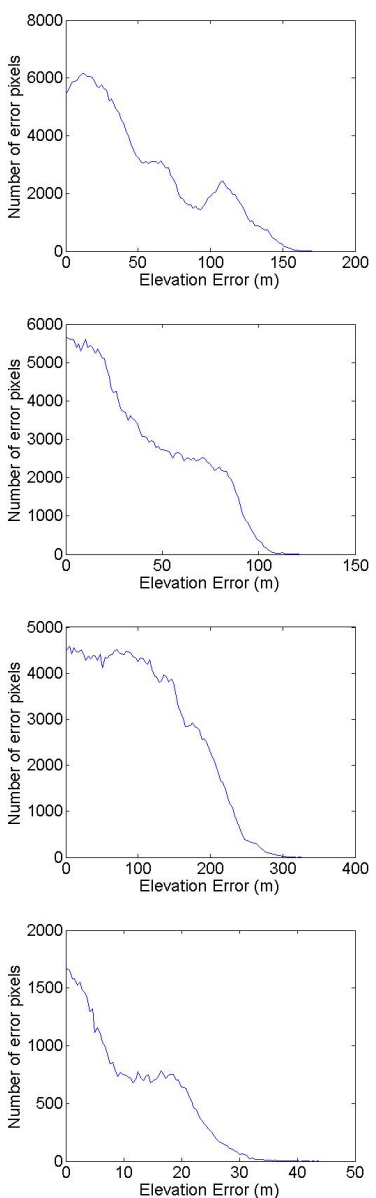

WM filter
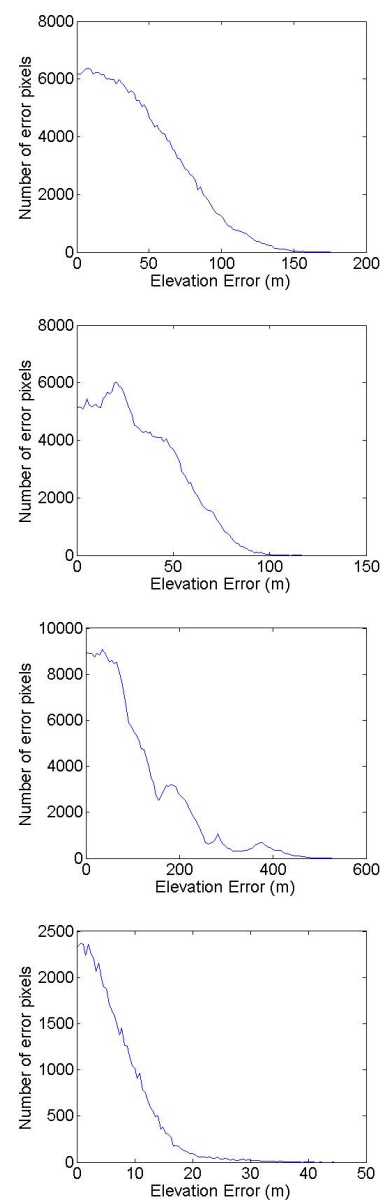

WInP filter
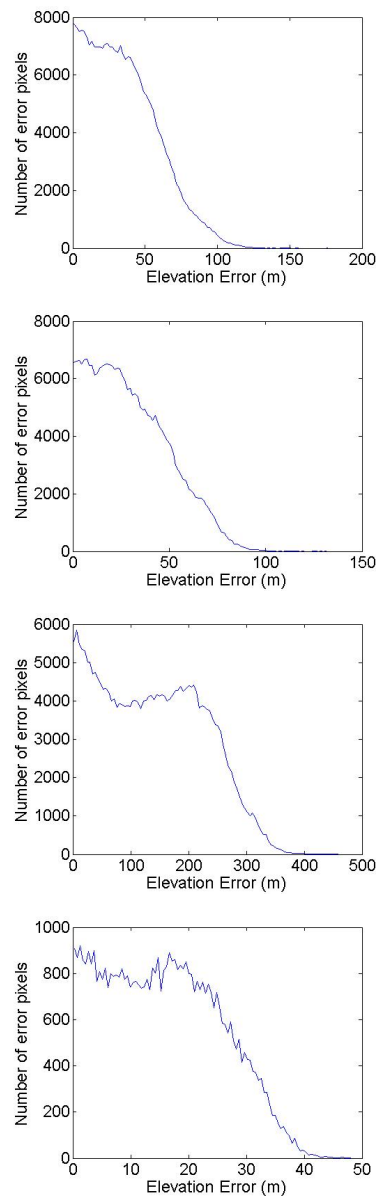

FAMM filter
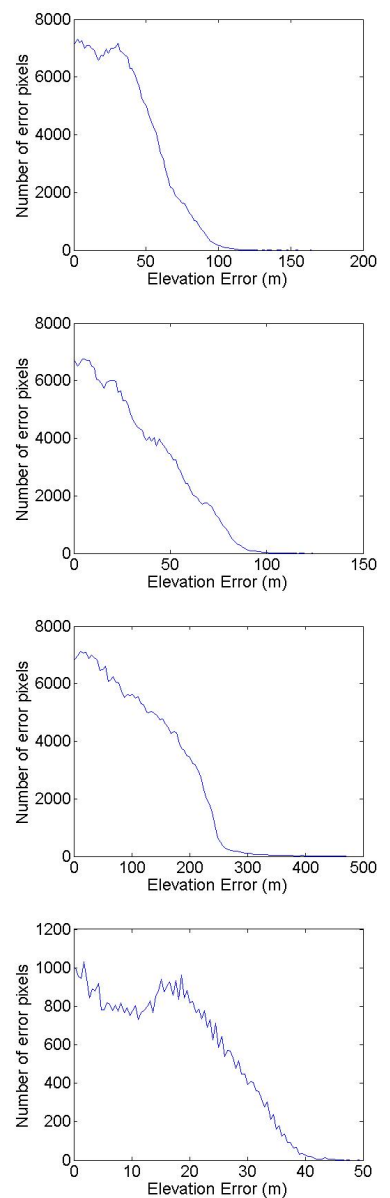

Figure 2: The distribution histogram of the elevation error between the DEM provided from ASTER satellite and the DEM produced from the filtered unwrapped phase of Radarsat-2 (first row), Envisat (second row), ERS-2 (third row) and COSMO-SkyMed (fourth row).

(Lopez-Martinez and Fabregas, 2002). Then the pixel that represents a signal coefficient in those 16 sub bands is located by using a generated mask defined based on the signal quality $\left(\Gamma_{\text {sig }}\right)$ and a threshold $\left(t h_{w}\right)$ parameters (Lopez-Martinez and Fabregas, 2002). After computing the noise mask using $t h_{w}$, only the real and imaginary part of signal coefficients are multiplied by 2 . Before applying the inverse wavelet transform, a new noise mask is calculated for each four sub signal bands by using logical OR. Finally, a noise mask for all image is obtained. This mask will be used to calculate the window size in the filtering step.

2.2.2 Filtering approach using a Coherence Map Mask The modified version of the López and Fabregas algorithm is presented in (Abdelfattah and Bouzid, 2008). This approach aim to overcome the inconsistencies introduced in the mask growing step presented in WInP filter. When doubling the dimensions of the merged mask, (Lopez-Martinez and Fabregas, 2002) complete the missing data by duplicating existing data. The modified filter as described below is distinguished from the WInP filter by two features (Abdelfattah and Bouzid, 2008):

- The inverse discrete wavelet transform (DWT) is applied using an adaptive mask extracted from the InSAR coherence map, sub-sampled to the convenient resolution of the corresponding wavelet decomposition level (to be processed).

- The threshold is adaptive, and is computed with respect to the four corresponding signal bands, and not the complete set of signal bands.
When generating the mask, the WInP algorithm considers the same threshold for all 16 signal bands. Because the approximations and the details coefficients are not the results of the same process, so they do not necessarily have the same signal dynamic. Thus, two thresholds are defined for each sub-band category. The values of these thresholds are computed with respect to the mean of the sub-band dynamics (Abdelfattah and Bouzid, 2008).

When doubling the dimensions of the merged mask to fit the previous scale band dimensions, the four pixels do not systematically classify by duplicating the corresponding pixel in the band of the current scale and these pixels do not have the same values. For this reason, (Abdelfattah and Bouzid, 2008) propose to generate a sub-sampled coherence map from the initial InSAR coherence map. The growing mask then depends on the coherence values of the four considered pixels in the band of the scale $2^{i-1}$ (Abdelfattah and Bouzid, 2008).

\section{PRESENTATION OF THE USED DATA}

In this paper we have used four pairs of SLC data from four different SAR radar systems. The first two pairs are provided from the Radarsat-2 and Envisat satellites respectively and captured over the zone of Mahdia in Tunisia. The others SLC pairs are given from ERS-2 and COSMO-SkyMed satellites and acquired over Ben Guerden in Tunisia too.

Table 1 describes the different acquisition mode features of each 


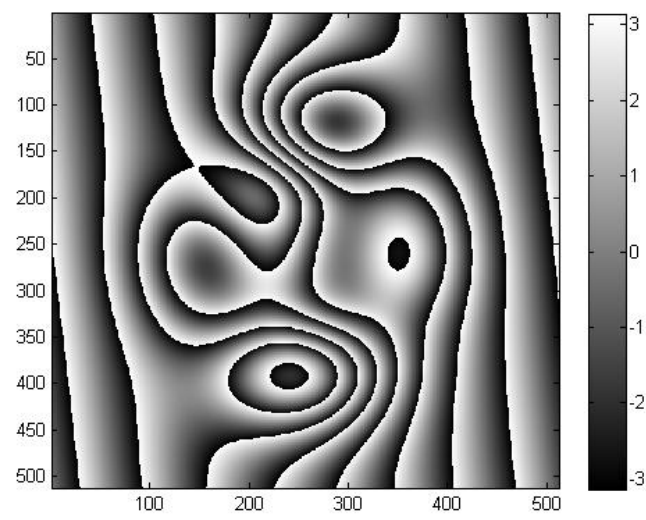

(a)

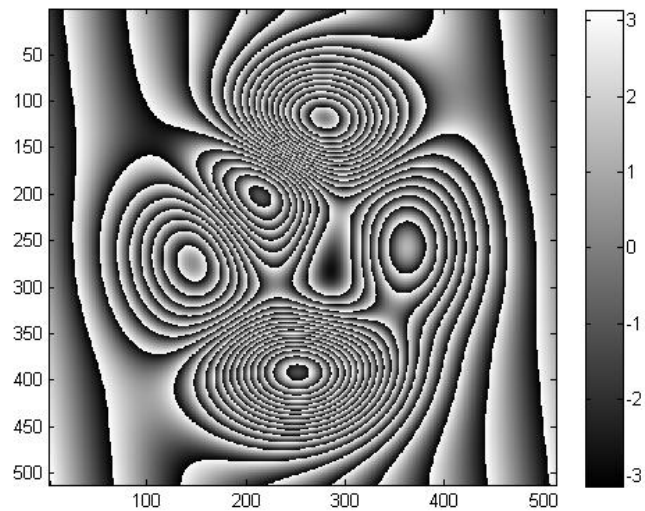

(c)

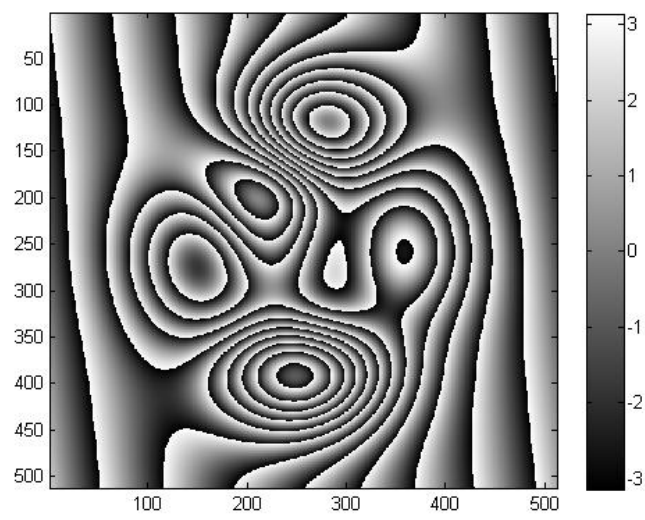

(b)

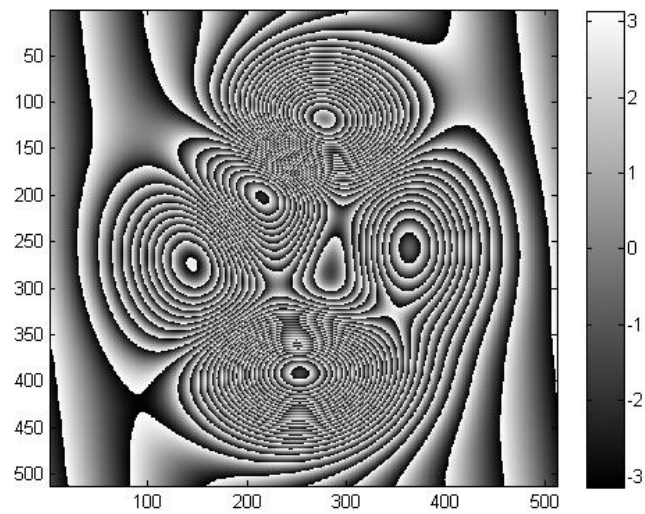

(d)

Figure 3: The simulated interferogram with different number of fringes.

satellite such as the incidence angle $\theta_{i}$, the coordinates of the acquired zone and the baseline between the two acquisitions:

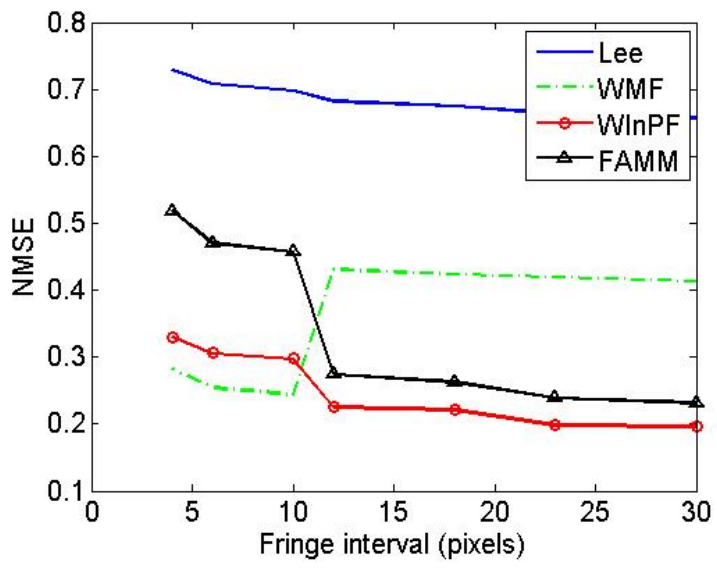

Figure 4: The relationship between the number of pixels between fringes and the NMSE of the simulated interferogram shown in Figure 3.

\section{EXPERIMENT RESULTS}

To validate the four filtering methods and compare the results, we used the interferograms produced by the SLC images from Radarsat-2, Envisat, ERS-2 and COSMO-SkyMed satellites and acquired over the regions of Mahdia and Ben Guerden in Tunisia

\begin{tabular}{|c|c|c|c|}
\hline Satellite & $\theta_{i}$ & Baseline $(\mathrm{m})$ & Coordinates \\
\hline Radarsat-2 & $28.03^{\circ}$ & 206.23 & $35.08-35.39 \mathrm{~N}$ \\
& $29.79^{\circ}$ & & $10.23-10.59 \mathrm{E}$ \\
\hline Envisat & $38.95^{\circ}$ & 376.01 & $34.64-35.71 \mathrm{~N}$ \\
& $42.72^{\circ}$ & & $10.23-11.22 \mathrm{E}$ \\
\hline ERS-2 & $18.61^{\circ}$ & 425.72 & $32.63-33.80 \mathrm{~N}$ \\
& $26.14^{\circ}$ & & $10.01-11.36 \mathrm{E}$ \\
\hline COSMO-SkyMed & $23.31^{\circ}$ & 25.53 & $33.04-33.14 \mathrm{~N}$ \\
& $24.21^{\circ}$ & & $10.94-11.07 \mathrm{E}$ \\
\hline
\end{tabular}

Table 1: Overview of the four satellites which their SLC data are used in this paper.

respectively. The filtering results of these approaches are illustrated in Fig 1. For the visual comparison, we consider that the filtered interferogram is well adapted to the unwrapping process if it respect the edges of the interferometric fringes and filter the homogeneous zones and it is clear that the filters operated in the wavelet domain (WInPF and FAMM) give better results than the spatial filters (Lee and WM filters).

\begin{tabular}{|c|c|c|c|c|}
\hline Interferogram & Lee & WMF & WInP & FAMM \\
\hline Figure 3 (a) & 0.6580 & 0.4137 & $\mathbf{0 . 1 9 6 8}$ & 0.2322 \\
\hline Figure 3 (b) & 0.6759 & 0.4250 & $\mathbf{0 . 2 2 1 8}$ & 0.2638 \\
\hline Figure 3 (c) & 0.7089 & $\mathbf{0 . 2 5 4 9}$ & 0.3058 & 0.4712 \\
\hline Figure 3 (d) & 0.7295 & $\mathbf{0 . 2 8 3 0}$ & 0.3304 & 0.5192 \\
\hline
\end{tabular}

Table 2: Normalized Mean Square Error (NMSE) comparaison between the four filtering methods applied to the different simulated interferograms of the Figure 3. 
Lee filter
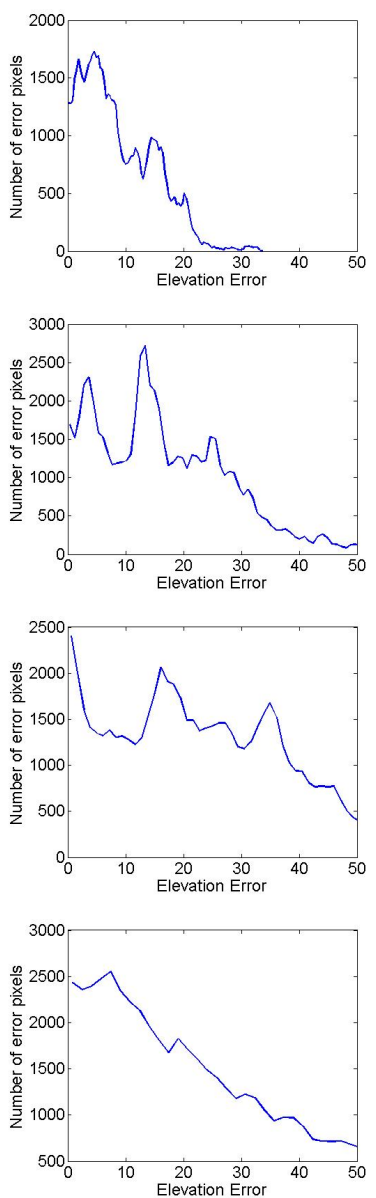

WM filter
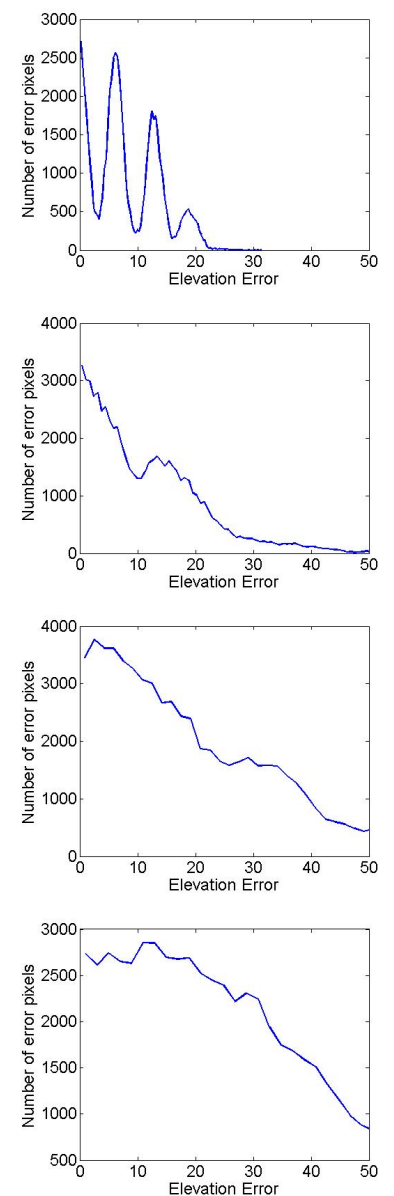

WInP filter
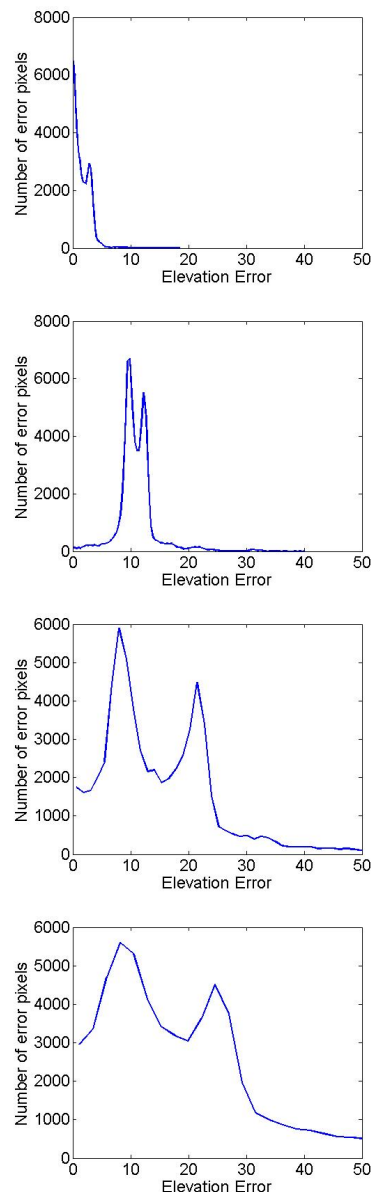

FAMM filter
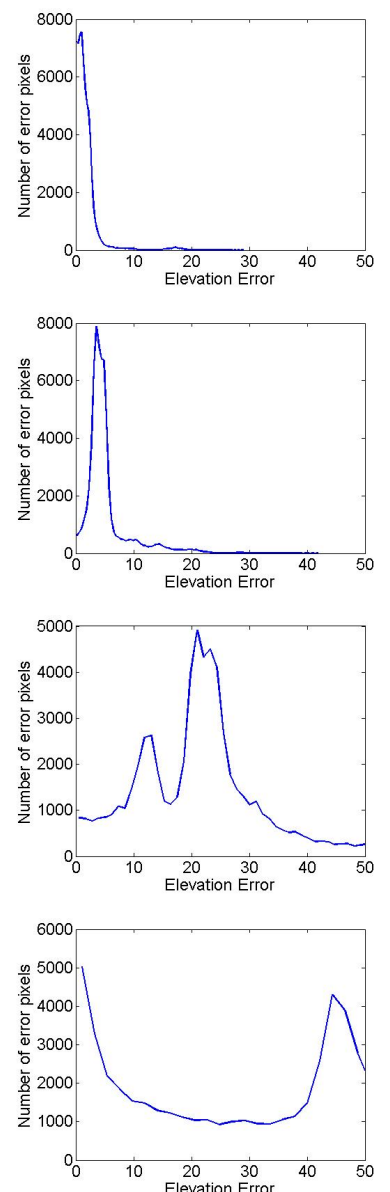

Figure 5: The distribution histogram of the elevation error between the original unwrapped phase image and the unwrapped filtered interferogramof Fig. 3. The first row: the interferogram of Fig. 3 (a), the second row: the interferogram of Fig. 3 (b), the third row: the interferogram of Fig. 3 (c) and the fourth row: the interferogram of Fig. 3 (d).

We then consider a quantitative comparison using statistical parameters. We compute the Normalized Mean Square Error (NMSE) the simulated interferogram filtered by Lee, the WMF, the WInP and the FAMM filters respectively and the original interferogram without noise. as shown in Table 2. From this table, we can notice that the filtering in the wavelet domain (such as the WInP and FAMM filters) is more adapted in the interferogram case. This can be justified by the fact that the wavelet transform is based on the extraction of the useful signal from the interferogram and by this way the edges of the fringes are keeped. The two filters in spatial domain (Lee and WMF), which are based on the compute of mean values, smooth the image by using the noisy and useful pixels without separation and this way gives a high error.

After computing the filtered InSAR image, we computed the unwrapped filtered real interferogram. To obtain the unwrapped phase, we used the Quality-Guided phase unwrapping algorithm described in (Zhong et al., 2011). After applying the unwrapping process, we computed the Digital Elevation Model (DEM) from the unwrapped filtered phase and compared it with the real DEM of the same acquired region. We used in this paper the ASTER Global DEM available in the Land Processes Distributed Active Archive Center website (http://gdex.cr.usgs.gov). The histograms of the absolute errors between the real DEMs, given from ASTER, and the obtained DEMs after unwrapping of the filtering phase are shown in Fig. 2. A simple visual comparaison between the four histograms for each satellite shows that in the case of the region of Mahdia the filters in the wavelet domain give lowest er- ror rate. But in the case of the region of Ben Guerden, the DEM etereen of the spatial filters are better. These results can be verified in the Table 3 representing the percentage of the number of pixels with DEM error lesser than 50 meters. In fact, the choice of the best interferogram filter depends on the width of the homogenius regions and the number of fringes in the interferograms. Then we can conclude that when the fringes are close to each other, it is recommended to apply the spatial filters. In the other case, when the fringes are so far from each other, the filters in wavelet domain should be used. This statement can be explained by the fact that the WInP and the FAMM filters use the DPWT with three decomposition levels. This means that the distance from each pixel of the third scale level band and its neighbour is $2^{3}=8$ pixels in the real interferogram (Lopez-Martinez and Fabregas, 2002), (Abdelfattah and Bouzid, 2008). This may cause error in the unwrapping process when the fringes are so close to each other because in this case, two neighbour pixels may be situated in two different fringes. To verify this assumption, we tested the four filters with a simulated interferogram with Matlab by wrapping a $3 D$ relief model generated by the meshgrid and peaks functions. This interferogram is $512 \times 512$ pixels size and having different number of fringes (Fig. 3). The four filtering approaches are tested with these simulated interferograms of the Figure 3 and then we compute the NMSE between the filtered phase images and the original one with respect to the fringe intervals (Figure 4). We also computed the interferograms elevation error between the filtered unwrapping phase and the original absolute phase image without noise. The Fig. 5 shows the relationship between 
the fringe pattern in the interferogram and those elevetion error of the four filters and we notice that when the fringes are close to each other and their number is high, the absolute elevation error grows in the case of the filters in wavelet domain and decreases when applying the filters in the spatial domain and this prouve our statement.

\begin{tabular}{|c|c|c|c|c|}
\hline Satellite & Lee & WMF & WInP & FAMM \\
\hline Radarsat-2 & 58.48 & 63.21 & 73.89 & $\mathbf{7 7 . 0 8}$ \\
\hline Envisat & 67.50 & 79.56 & $\mathbf{8 2 . 4 3}$ & 80.25 \\
\hline ERS-2 & 25.76 & $\mathbf{3 2 . 0 2}$ & 21.12 & 27.79 \\
\hline COSMO-SkyMed & 100 & $\mathbf{1 0 0}$ & 100 & 100 \\
\hline
\end{tabular}

Table 3: The percentage of the number of pixels with DEM error lesser than 50 meters with respect to the total number of DEM error pixels for the four different satellites.

\section{CONCLUSIONS AND FUTURE WORK}

In this paper we presented a comparative study between interferogram filtering algorithms in spatial and wavelet domains. With the experimental results, we finished by concluding that the multidimensional methods, and in particular the approaches based on the wavelet transfrom, give the best results generaly but when the fringes of the interferogram are so close to each other, the filters in the spatial domain are more adapted and give best unwrapping results. This result is verified with simulated and real interferograms generated from four different SAR systems and may be used to develop a new InSAR filtering approach combining the filters in the wavelet and spatial domains.

\section{ACKNOWLEDGEMENTS}

The authors would like to thank the Canadian Space Agency for offering us the Radarsat- 2 data within the context of the SOAR-E 5088 project.

\section{REFERENCES}

Abdelfattah, R. and Bouzid, A., 2008. Sar interferogram filtering in the wavelet domain using a coherence map mask. In: ICIP 2008. 15th IEEE International Conference on, IEEE, pp. 18881891.

Abdelfattah, R. and Nicolas, J., 2002. Topographic sar interferometry formulation for high-precision dem generation. Geoscience and Remote Sensing, IEEE Transactions on 40(11), pp. 2415-2426.

Arce, G., 1998. A general weighted median filter structure admitting negative weights. Signal Processing, IEEE Transactions on 46(12), pp. 3195-3205.

Bezerra Candeias, A., Mura, J., Dutra, L. and Moreira, J., 1995. Interferogram phase noise reduction using morphological and modified median filters. In: IGARSS'95, International, Vol. 1, IEEE, pp. 166-168.

Fornaro, G. and Guarnieri, A., 2002. Minimum mean square error space-varying filtering of interferometric sar data. Geoscience and Remote Sensing, IEEE Transactions on 40(1), pp. 11-21.

Geudtner D., S. M. and R., W., 1994. Sar-interferometry with ers-1 data. In: SAR-interferometry with ERS-1 data, PIERS.

Goldestein, R., Zebker, H. and Werner, C., 1988. Satellite radar interferometry- two-dimensional phase unwrapping. Radio Science 23(4), pp. 713-720.
Hess-Nielsen, N. and Wickerhauser, M., 1996. Wavelets and time-frequency analysis. Proceedings of the IEEE 84(4), pp. 523540.

Huot, E., Rudant, J.-P., Classeau, N., Flasque, B., Guillope, P., Herlin, I. L., Sigelle, M. and Stroobants, W., 1998. Image processing for multitemporal sar images. SAR Image Analysis, Modeling, and Techniques (198), pp. 198-209.

Lee, J., Papathanassiou, K., Ainsworth, T., Grunes, M. and Reigber, A., 1998. A new technique for noise filtering of sar interferometric phase images. Geoscience and Remote Sensing, IEEE Transactions on 36(5), pp. 1456-1465.

Lopez-Martinez, C. and Fabregas, X., 2002. Modeling and reduction of sar interferometric phase noise in the wavelet domain. Geoscience and Remote Sensing, IEEE Transactions on 40(12), pp. 2553-2566.

Xie, X. and Pi, Y., 2011. Phase noise filtering and phase unwrapping method based on unscented kalman filter. Systems Engineering and Electronics, Journal of 22(3), pp. 365-372.

Zhong, H., Tang, J., Zhang, S. and Chen, M., 2011. An improved quality-guided phase-unwrapping algorithm based on priority queue. Geoscience and Remote Sensing Letters, IEEE 8(2), pp. 364-368. 